\title{
SGK1 Inhibits ULK2 Autophagy Activity
}

\author{
Sung Hwa Shin ${ }^{1}$, Eun Jeoung Lee ${ }^{1}$, Sunghee Hyun ${ }^{2}$, Sang Sun Kang ${ }^{*}$ \\ ${ }^{1}$ Department of Biology Education, Chungbuk National University, Cheongju, Korea \\ ${ }^{2}$ Department of Biomedical Laboratory Science, Eulji University, Daejeon, Korea \\ Email: ^jin95324@cbu.ac.kr
}

How to cite this paper: Shin, S.H., Lee, E.J., Hyun, S. and Kang, S.S. (2020) SGK1 Inhibits ULK2 Autophagy Activity. American Journal of Molecular Biology, 10, 12-28. https://doi.org/10.4236/ajmb.2020.101002

Received: July 16, 2019

Accepted: November 10, 2019

Published: November 13, 2019

Copyright $\odot 2020$ by author(s) and Scientific Research Publishing Inc. This work is licensed under the Creative Commons Attribution International License (CC BY 4.0).

http://creativecommons.org/licenses/by/4.0/

\begin{abstract}
Serum- and glucocorticoid-induced kinase 1 (SGK1) is known to have consensus sequence of phosphorylation site $\mathrm{R}-\mathrm{x}-\mathrm{R}-\mathrm{x}-\mathrm{x}-(\mathrm{S} / \mathrm{T})-\Phi$, where $\Phi$ is any hydrophobic amino acid and arginine residues are conserved at positions -5 and -3 relative to positions of Ser/Thr residues that are phosphorylated in the presence of SGK1. UNC-21-like kinase 2 (ULK2) also harbors putative SGK1 phosphorylation sites at both Ser507 $\left({ }^{502} \mathrm{RsRnsSG}^{508}\right)$ and Ser750 $\left({ }^{745} \mathrm{RtRttSV}{ }^{751}\right)$ residues. Thus, the objective of this study was to determine whether Ser507 and Ser750 residues of ULK2 could be phosphorylation sites of SGK1 as one of its authentic substrate proteins. Using ULK2 507 and 750 serine residue un- or phosphorylation analog (S507AS750A or 507DS750D), we observed that modification of Ser507 or Ser750 residue was required to activate the kinase activity of ULK2 and sensitize ULK2 to stress or starvation while simultaneously enhancing its active state and autophagy characteristics, suggesting that phosphorylation at Ser750 or Ser507 residue could modulate its subcellular localization and protein interaction with AMPK1 $\alpha$ to activate ULK2. We also observed that ULK2 autophagy activity was enhanced by GSK650394 (an SGK1 inhibitor) to compensate survival capacity through increasing its association with LC3 and phosphorylation. When SGK1 known to be associated with cell survival was inhibited by GSK650394, ULK2 autophagy pathway was activated to avoid cell death alternatively. Thus, our observations indicate that phosphorylation of ULK2 by SGK1 can regulate cell survival as an alternative modulation of ULK2 functions.
\end{abstract}

\section{Keywords}

ULK-2, SGK1, Phosphorylation, Autophagy, Cell Survival, Signal Transduction

\section{Introduction}

Uncoordinated 51-like kinase 2 (ULK2), like ULK1, is a member of the serine/threonine kinase protein family that plays an essential role in the regulation 
of autophagy in mammalian cells. ULK2 is expressed ubiquitously [1] [2] [3] [4]. Its role seems to be redundant with that of ULK1. Although specific functions of ULK1 and ULK2 in autophagy are currently unclear, ULK2 can compensate for deletion of ULK1 [5] [6].

ULK2 functions in many fundamental biological processes, including cell fate determination, metabolism, transcriptional control, and autophagy [4] [5] [6]. The key role of autophagy in normal cellular homeostasis and multiple diseases suggests that mechanistic insights into autophagy could drive the development of novel therapeutic approaches [1] [2] [3]. Together with ULK1, ULK2 plays a central role in the autophagy signaling pathway [7] [8]. However, recent studies have suggested that the activity of ULK2 must be carefully regulated by mechanisms that are individually modulated for each substrate to avoid indiscriminate phosphorylation by ULK1 [9] [10].

Although the mechanism that regulates ULK2 in the autophagic process is not fully understood yet, precise control seems to be achieved through a combination of phosphorylation, localization, and interactions with ULK2 binding proteins [7] [8] [9] [10].

Unlike ULK1 that is predominantly found in the cytosol, ULK2 is mainly located in the nucleus, although it is also found in the cytosol. Recently, the mechanism by which ULK2 is localized to the nucleus has been reported [11].

ULK2 does not have any recognizable short, basic, classic import, or export sequences. It contains PY-NLS sequences which contribute to its nuclear localization [11]. This character is a distinguishable feature from ULK1. Thus, its localization is likely to be indirectly regulated through association with binding proteins. It has been suggested that a binding protein can regulate the subcellular localization of ULK2 by inhibiting its nuclear export [11].

Serum- and glucocorticoid-induced kinase 1 [SGK1] is a Ser/Thr protein kinase that is transcriptionally regulated by serum and/or glucocorticoids in mammary epithelial cells and Rat-2 fibroblasts [12] [13] [14]. SGK1 is also transcriptionally regulated by corticosteroids in several types of cells. SGK1 has been identified as a protein that can promote cell survival. It has the same function as Akt kinase [12]-[17].

Many SGK1 substrates have been characterized thus far [15] [16] [17]. In concert with SGK1, they appear to play a role in propagating effects of PI3K, including promotion of cell-cycle progression and autophagy consequently [17]. Many physiological SGK1 substrates remain to be characterized [12] [13]. The manner in which they are modulated in the presence of SGK1 also remains unclear. Consensus SGK1 substrate site has been characterized as $\mathrm{R}-\mathrm{x}-\mathrm{R}-\mathrm{x}-\mathrm{x}-[\mathrm{S} / \mathrm{T}]-\Phi$ motif, where $\Phi$ is any hydrophobic amino acid and arginine residues are conserved at positions -5 and -3 relative to positions of Ser/Thr residues that are phosphorylated in the presence of SGK1 [12]-[17]. Since the substrate motif of SGK1 is similar to that of Akt1, proteins containing such amino acid sequences seem to be also potent Akt1 substrates [14].

Ser507 $\left({ }^{502} \mathrm{RsRnsSG} \mathrm{G}^{508}\right)$ and Ser750 $\left({ }^{745} \mathrm{RtRttSV}{ }^{751}\right)$ of ULK2 were found to have 
consensus SGK1 substrate sequences $\{\mathrm{R}-\mathrm{x}-\mathrm{R}-\mathrm{x}-\mathrm{x}-[\mathrm{S} / \mathrm{T}]-\Phi\}[4]$. Thus, the objective of this study was to determine whether SGK1 could phosphorylate Ser507 or Ser750 residue of ULK2 as one of its specific substrate proteins [4] [14]. We found that SGK1-mediated modification of Ser750 residue of ULK2 promoted its nuclear localization and autophagy activity with a synergic effect on its functional interactions with AMPK1 $\alpha$ [18]. In addition, we found that ULK2 expression was enhanced by treatment with GSK650394 (a SGK1 specific inhibitor) to compensate survival capacity by increasing its association with LC3. In the case of activation of SGK1 [known to be associated with cell survival] by insulin treatment, ULK2 autophagy pathway was inhibited through phosphorylation of Ser507 or Ser750. Our results suggest that ULK2 autophagy seems to be an alternative cell survival pathway when PI3-PDK1-SGK1 pathway is inhibited.

\section{Materials and Methods}

Site-Directed Mutagenesis-Human ULK2 cDNA was obtained from Korea Human Gene Bank [Gene ID; KIAA0623] and coned into pEGFP1 vector [Clontech, Palo Alto, CA, USA]. In order to obtain mutants, amino acid changes were introduced using mutated oligonucleotides for S/A [up 5'-agg gat cgttggGcc Gcggtggtg ccc cgc gta-3', down 5'-gcggggcaccaccgCggCccaacgatccet acg-3'] or S/D [up 5'-agg gat cgttggGAc GAC gtggtgccccgcgta-3', down 5'-gcggggcaccac GTC gTCccaacgatccct acg-3'] and wild-type ULK2 as template. These ULK2 mutant constructs were obtained using QuikChange ${ }^{\circledR}$ XL Site-Directed Mutagenesis Kit (Stratagene). All ULK2 mutants were confirmed by DNA sequencing. Human embryonic kidney (HEK 293) cells were transfected with ULK2 or mutant constructs as described previously.

\subsection{Western Blotting}

Cells transfected with cDNA-containing plasmids were grown in cell culture plates until $48 \mathrm{~h}$ after transfection. Cells were rinsed three times with PBS. The buffer solution was gently removed and cells were lysed in PBS containing 1\% Triton X-100 and protease inhibitor mixtures [Thermo Scientific]. To test the reversibility of covalent dithiaarsanane adducts, we included $10 \mathrm{~mm}$ mercaptoethanol in the lysis buffer. The lysate was resolved on $8 \%$ non-reducing SDS-polyacrylamide gel, which was transferred to an Immobilon membrane (Millipore). The membrane was incubated in blocking buffer (Tris-buffered saline with $0.05 \%$ Tween 20 with 5\% nonfat milk) containing each antibody (at 1:1000 dilution, Covance, Inc.) at $4^{\circ} \mathrm{C}$ overnight. The antigen-antibody complexes were visualized using a secondary goat-anti-rabbit HRP-conjugated antibody at 1:100,000 dilution (Thermo Scientific).

Measurements of Autophagy-To measure autophagy, we used LC3B western blot (Bio-Protocol Com) method following the manufacture' guide. Biochemical methods such as western blot to measure autophagic proteins have been used in autophagy studies. Total amount of microtubule-associated protein light chain 3 
(LC3), the mammalian homologue of autophagy-related Atg8 in yeast, was used as a tool in autophagy. Western blot was performed as the same as the above "Immunoblotting" section with antibody against LC3. Each assay was performed in five replicates.

Solutions and drugs-Fluo4-AM, wortmannin, insulin, and AMPK1 were purchased from Sigma [St. Louis, MO, USA]. Stock solutions were initially prepared in dimethyl sulphoxide [DMSO] at a concentration of $1 \mathrm{mM}$ and then stored at $-20^{\circ} \mathrm{C}$. The final DMSO concentration in the experimental bath solution containing phorbolesters never exceeded $0.5 \%$.

FACS-EGFP ULK2 WT, ULK2 S507A, S507AS750A, and S507DS750D mutant, and EGFP vector were transfected into cells and rates of apoptosis measured with an Annexin V-PE apoptosis detection kit I (BD Biosciences, USA). Transfected cells were washed twice with cold $\mathrm{PBS}$ and resuspended in binding buffer (0.01 M Hepes/NaOH [pH 7.4] 0.14 M NaCl, $2.5 \mathrm{mM} \mathrm{CaCl}_{2}$ ). After transferring $1 \times 10^{5}$ cells in $100 \mathrm{ml}$ to a $5 \mathrm{ml}$ culture tube, $5 \mathrm{ml}$ of Annexin V-PE and 5 $\mathrm{ml}$ of 7-Amino-actinomycin $\mathrm{D}$ were added to the tube. These cells were vortexed gently and incubated at $25^{\circ} \mathrm{C}$ for $15 \mathrm{~min}$ in the dark. After adding $400 \mathrm{ml}$ of binding buffer to each tube, FACS was performed within $1 \mathrm{hr}$ on a Coulter Epics Elite equipped with a gated amplifier to give enhanced system performance in the Core Facility of Chungbuk National University.

\subsection{Confocal Microscopy}

HEK293 cells were seeded overnight at $60 \%$ confluence onto culture slides coated with human fibronectin [Becton Dickinson, MA] in the $5 \% \mathrm{CO}_{2}$ cell growth incubators. The cells were washed several times with ice-cold PBS and fixed in 3\% paraformaldehyde for 10 minutes. The fixed cells were permeabilized with $0.1 \%$ Triton X-100 for 10 minutes and blocked for 1 hour in PBS containing $5 \%$ BSA [Sigma, USA] and $0.1 \%$ Tween. Following incubation with a polyclonal antibody against EGFP, ULK2 or monoclonal antibody against ULK2, actin, LC3, or AMPK1 $\alpha$, the cells were washed and stained further with a conjugated donkey anti-rabbit IgG prior to processing the slides for immunofluorescence. After an additional 20 minutes of incubation at $37^{\circ} \mathrm{C}$, the cells were fixed, permeabilized, and decorated with either an anti-ULK2 actin, LC3, or AMPK1 $\alpha$ antibody. As a secondary antibody, Alexa Fluor 568-conjugated donkey anti-rabbit or Fluor 488-conjugated goat anti-mouse (Molecular Probes, Inc., Eugene, OR) was used. Confocal microscopy analysis was performed with LSM710 (Zeiss, Germany) at the Center for Experimental Research Facilities of Chungbuk National University. Cell surface area was also determined with the confocal microscopy analysis. Fraction of nuclear localization and cytoplasmic localization $(\mathrm{Fc} / \mathrm{n})$ and Pearson's correlation coefficient (PCC) were determined with the confocal microscopy analysis program provided by the manufacture [11].

Statistics-Data are expressed as means \pm standard error of the mean (SEM). Statistical significance was determined via Student's t-test or Welch's t-test. Sta- 
tistical significance was considered when $p$ value was less than 0.05 .

\section{Results}

\subsection{Interaction of ULK2 with LC3 Is Enhanced by SGK1 Inhibitor Treatment}

To elucidate the biological significance of ULK2 phosphorylation by SGK1, we attempted to find an endogenous ULK2 property change depending on SGK1 activator or inhibitor (Figure 1). Because LC3 binding motifs were also found in ULK2 S/P spacer $\left({ }^{359}\right.$ tddfvlvphni ${ }^{360}$; see Figure $2(\mathrm{~b})$ ), we also determined whether endogenous ULK2 formed a protein complex with LC3 in HEK293 cells [4]. As shown in Figure 1, ULK2 immunoprecipitants contained both LC3I and LC3II in HEK293 cells. Antibodies against ULK2 successfully captured LC3I and LC3II from the same lysate (lane 3). As a negative control, ULK2 not containing LC3-interacting region (LIR) motifs (W/F/Y)XX(L/I/V) was used , and there was no LC3 signal (lane 1). Unexpectedly, GSK650394 treatment enhanced the binding between LC3II and ULK2 to be five times higher than insulin treatment or non-treatment (glucose free for $12 \mathrm{hr}$ ). To monitor ULK2 protein expression, ULK2 rabbit antibody was used for western blot (Figure 1 upper row). Insulin treatment also increased ULK2 expression (lane 3) to be 3 times higher than

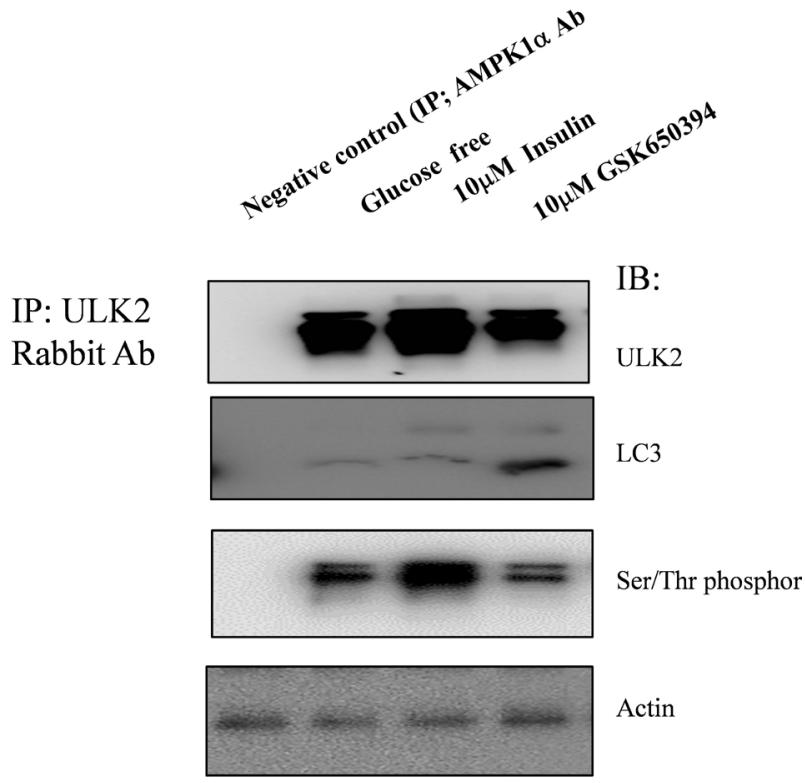

Figure 1. HE293 cell autophagy activity in response to insulin or GSK650394 treatment. HEK293 cells were exposed to $1 \mu \mathrm{M}$ insulin or $300 \mathrm{nM}$ GSK-650394 (SML0773 Sigma) in glucose free DMEM for $12 \mathrm{hr}$. After ULK2 immunoprecipitation with the mouse ULK2 antibody the expression ULK2 was measured with a western blot with Rabbit ULK2, anti-phosphor-Ser/thr, LC3, or actin Ab (from Cell Signaling Technology) was performed. As the negative control for the immunoprecipitation, AMPK $1 \alpha$ Ab was used. For the cell amount control, the western blot with actin Ab was shown. The autophagy activity which was detected by LC3 Ab western (third lane) was 5 times enhanced by the specific SGK1 inhibitor (GSK-650394) treatment [19] than by the insulin or no treatment control. This is one of four repeated experiment results. 
(a)

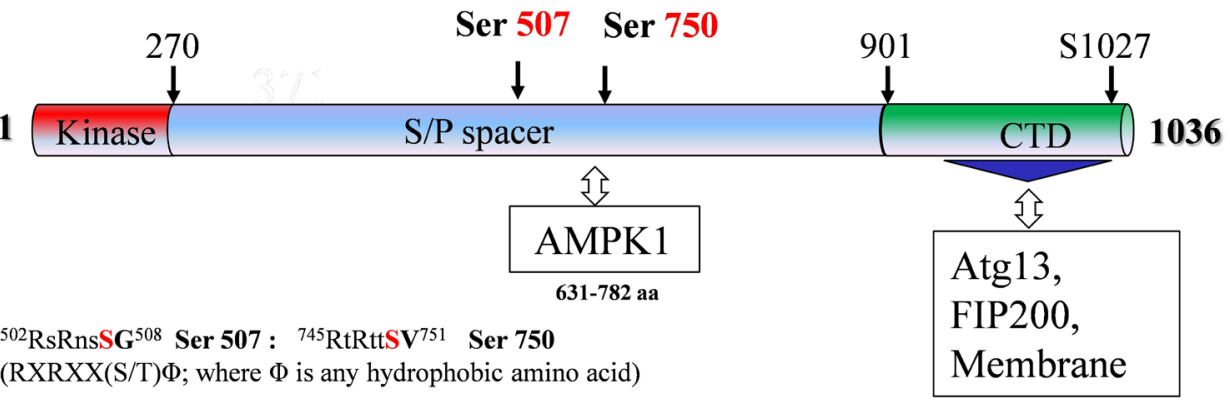

${ }_{\text {(b) }}{ }^{502} \mathrm{RsRnsSG}^{508}$ Ser 507 : ${ }^{745} \mathrm{RtRttSV}^{751}$ Ser 750
$(\mathrm{RXRXX}(\mathrm{S} / \mathrm{T}) \Phi$; where $\Phi$ is any hydrophobic amino acid)

10

IB:

(c)

IP:

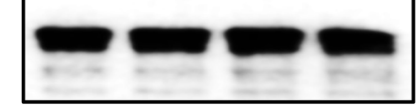

ULK2

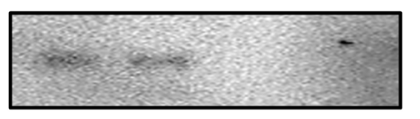

Ser/Thr phosphor

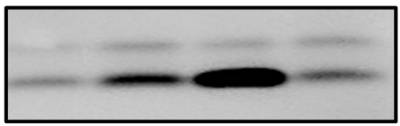

LC3

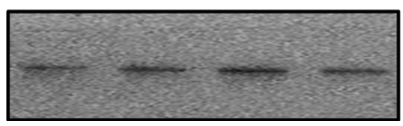

Actin

(d)

DAPI

ULK2 WT

LC3

Merge
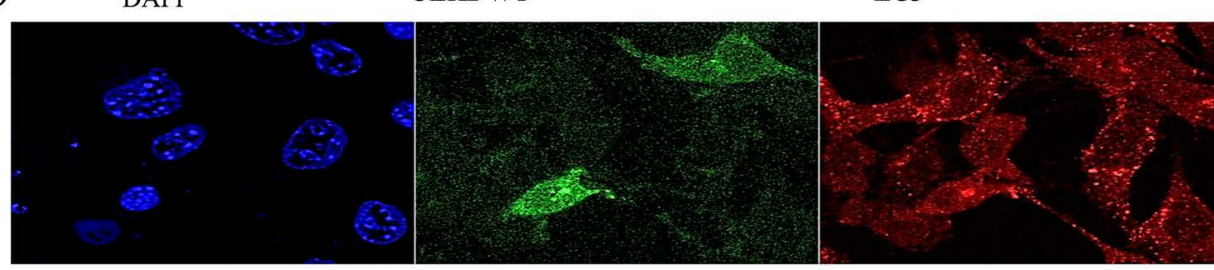

$\mathrm{Fn} / \mathrm{c}: 0.34+/-0.06(\mathrm{~N}=5)$

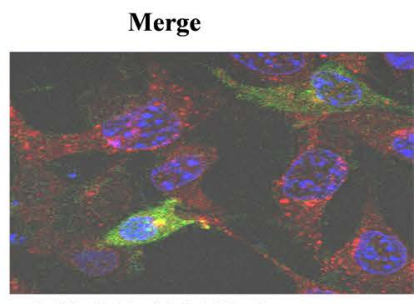

PCC: $0.58+/-0.06(\mathrm{~N}=5)$

\section{ULK2 S507AS750A}
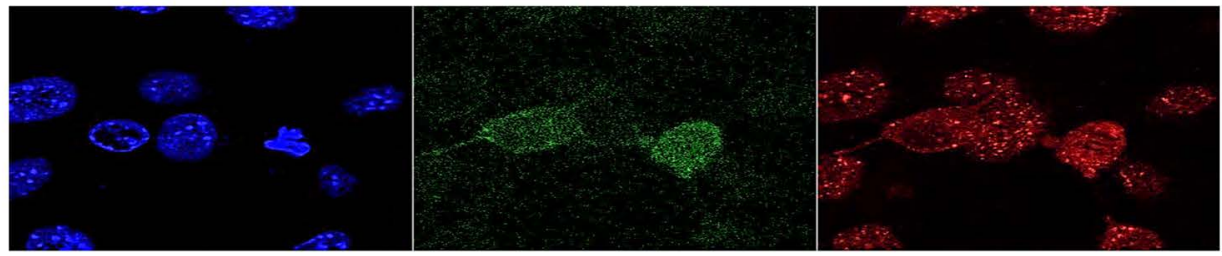

Fn/c: $0.22+/-0.03(\mathrm{~N}=5)$

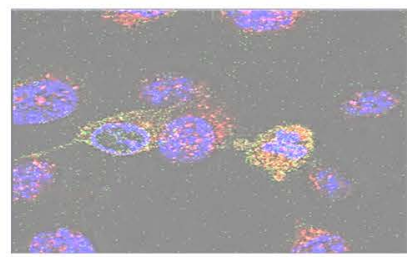

PCC: $0.89+/-0.06(\mathrm{~N}=5)$

\section{ULK2 S507DS750D}
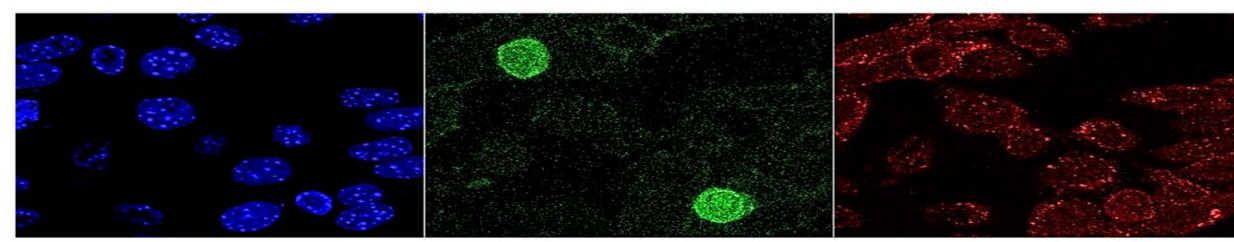

$\mathrm{Fn} / \mathrm{c}: 0.43+/-0.6(\mathrm{~N}=5)$

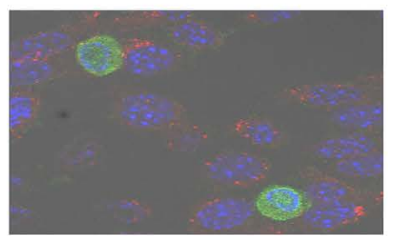

PCC: $0.32+/-0.03(\mathrm{~N}=5)$

Figure 2. The phosphorylation on (Ser507 and/or 750) of ULK2 by SGK1. (a) The mouse ULK2 (1036aa length) topology. From the $\mathrm{N}$ terminal, it contains the protein kinase (kinase), serine-proline rich (S/P), and $\mathrm{C}$ terminal domain (CTD; 901 - 1036 aa) 
which interacts with the membrane, Atg13, and FIP200. The putative AMPK1 binding site (631 - 782 aa), and the putative SGK1 phosphorylation sites (both Ser507 in ${ }^{502} \mathrm{RsRnsSg}^{508}$ and Ser750 in ${ }^{745} \mathrm{RtRttSv}{ }^{751}$ ) locate in the S/P space domain where the putative AMPK1 binding region (631 - $782 \mathrm{aa}$ ) is overlapped. The above arrow points to phosphorylation protein by SGK1. (b) The alignment of ULK2 WT (Gene ID; KIAA0623), S507A, S507D, S750D or S750A with the consensus SGK1 substrate motif. The putative SGK1 phosphorylation site (Ser507 and 750) of ULK2 is located in the specific conserved SGK1 substrate motif $\{\mathrm{R}-\mathrm{R}-\mathrm{X}-[\mathrm{S} / \mathrm{T}]-\Phi\}$. With the site directed mutagenesis, S507A S750A and S507D S750D were constructed and aligned WT and SGK1 substrate specific motif. (c) The phosphorylation of Ser507 $\left({ }^{502} \mathrm{RsRnsSg}^{508}\right)$ or the Ser750 residues $\left({ }^{745} \mathrm{RtRttSv}{ }^{751}\right)$ of ULK2 in HEK293 cells. The host cells were transiently transfected with EGFP-ULK2 WT, S507A, S507AS750A, or S507DS750D plasmid. After 48 h, the cells were lysed, total proteins were recollected, and immunoprecipitation was conducted with EGFP monoclonal mouse antibody. Western blot assays were then conducted with a rabbit ULK2 Ab or an anti-phosphor Ser/Thr phosphorylation specific antibody in order to confirm whether Ser507 or Ser750 residue of ULK2 could be phosphorylated by SGK1 in HEK293 cells [second lane] The autophagy activity of ULK2 WT or mutants was measured with the antibody against LC3 as manufacture' recommendation (third lane). The western blot with actin Ab was shown as the cell amount control (forth lane). This is one of four repeated experiment results. (d) Confocal microscopic analysis of transfected EGFP-ULK2 WT or mutants (S507A S750A or S507D S750D) (all constructs are shown in green) with LC3 (red) to access each autophagy activity. The transfected EGFP ULK2 (S507A S750A) with LC3 was merged with the high PCC $(0.89 \pm 0.06 ; \mathrm{N}=5)$ value as yellow color. However, the transfected EGFP-ULK2 S507D S750D (PCC was $0.32 \pm 0.03 ; \mathrm{N}=5$ ) was not merged well with LC3 (shown in red), even though PCC between LC3 and ULK2 WT was $0.88 \pm 0.06(\mathrm{~N}=5)$. The PCC value was matched with the western bolt results (Figure $3(\mathrm{c})$ third lane). Fn/c value of EGFP-ULK2 S507DS750D (0.45) was the highest among them. DAPI staining represents the nuclear region (blue). Fn/c value and PCC were present under the picture.

GSK650394 treatment (lane 4) or non-treatment (lane 2). To determine serine phosphorylation of ULK2 protein, a phosphor serine threonine rabbit antibody was utilized for western blot. Results are shown in Figure 1 third row. GSK650394 treatment (lane 4) decreased ULK2 serine phosphorylation 3 times than no treatment (lane 2), while insulin treatment (lane 3) increased ULK2 serine phosphorylation 5 times than no treatment. Anti-actin Ab was used as internal control in Figure 1 forth row. Taken together, our results support the hypothesis that the association of ULK2 with LC3II is regulated antagonistically, depending on the phosphorylation of serine residue of ULK2 by SGK1.

\subsection{Phosphorylation at Both Ser507 ( ${ }^{502} R s R n s S G{ }^{508}$ ) and Ser750 ( ${ }^{745} \mathrm{RtRttSv}^{751}$ ) of ULK2 by SGK1}

ULK2 contributes to a variety of important functions including autophagy. The regulation of ULK2 has been shown to occur via posttranslational modifications (phosphorylation, glycosylation, and ubiquitination) and protein-protein interactions, including adenosine monophosphate-activated kinase 1 (AMPK1) and Atg13-FIP200 (Figure 2(a)) [1] [2] [3] [4]. We recognized putative SGK1 phosphorylation sites at Ser507 and Ser750 residues of ULK2 with consensus sequence of R-X-R-X-X-[S/T]- $\Phi$ (Figure 2(a) and Figure 2(b)) [4]. Thus, we evaluated phosphorylation of both residues and their biological significance. We subsequently attempted to pinpoint the target Ser residue for phosphorylation of Ser507 and/or Ser750 of ULK2 by SGK1. Several studies have documented that both Ser residues within the consensus motif are subjected to phosphorylation. Therefore, we focused on both Ser750 and Ser507.

To confirm our hypothesis that SGK1 phosphorylates S507 and S750 residues, we constructed double mutant (ULK2 S507AS750A, unphosphorylated form; 
and ULK2 S507D S750D, phosphorylated form) with the site directed mutagenesis. To confirm that SGK1 could phosphorylate ULK2, we conducted western blotting for immuno-purified ULK2 from HEK293 cells using an anti-phosphor Ser/Thr specific antibody as described in the Materials and Methods section. As shown in Figure 2(c) second row, phosphorylation of ULK2 by SGK1 was only detected with Ser/Thr phosphor Ab on ULK2 WT and S507A (second row first and second lanes). Thus, the result suggests that 5507 and S750 residues of ULK2 are authentic phosphorylation sites of SGK1. Thus, ULK2 is one specific substrate of SGK1.

Next, we compared LC3II binding with ULK2WT, ULK2 S507A, S507AS750A, or S507DS750D mutant to obtain more information on the role of Ser507 and Ser750 phosphorylation. Our results in Figure 2(c) third row showed that ULK2 S507AS750A bound to LC3II more strongly than WT or S507DS750D, suggesting that ULK dephosphorylation at Ser507 and 750 residue could increase its binding to LC3II. Because LC3II binding indicates its autophagy activity, ULK2 S507A S750A mutant seems to contain more autophagy activity than ULK2 S507D S750D mutant or WT. EGFP ULK2 expression level was then monitored with an anti-ULK2 rabbit Ab (Figure 2(c) first row). Consistent with Figure 1 results, unphosphorylated ULK2 (S507AS750A mutant) obtained more its LC3II binding ability than phosphorylated ULK2 (S507DS705D mutant), suggesting that SGK1 phosphorylation on ULK2 could inactivate its autophagy activity.

To access each autophagy activity visually, we conducted confocal microscopic analysis of transfected EGFP-ULK2 WT or mutants (S507AS750A or S507DS750D) (all constructs are shown in green) with LC3 (red). The transfected EGFP ULK2 (S507A S750A) with LC3 was detected with the highest PCC $(0.89 \pm 0.06(\mathrm{~N}=5)$ value as yellow color (Figure 2(d) middle row right), while the transfected EGFP-ULK2 S507D S750D was shown the low PCC value $(0.32 \pm 0.03 ; \mathrm{N}=5)$ was not colocalized well with LC3 (shown in red), even though PPC between LC3 and ULK2 WT was $0.58 \pm 0.06(\mathrm{~N}=5)$. The high PCC value is correlated with the high autophagy activity in the western bolt results (Figure $3(\mathrm{c})$ third lane). Together, the confocal result and Western blot result suggest that the phosphorylation of ULK2 Ser507 and Ser750 residue by SGK1 seems to inhibit its autophagy activity.

To determine whether Ser507 Ser and 750 residue change of ULK2 might be attributable to its subcellular localization or expression-level deviation, we also measured the Fraction ratio between nuclear and cytoplasm (Fn/c) value of each ULK construct under the confocal microscopy. EGFP-ULK2 S507DS750D (0.45) was the highest among three (ULK2 WT, S507AS750A, and S507DS750D), suggesting that the phosphorylation on ULK2 Ser507 and Ser750 residue by SGK1 promotes its nuclear localization.

\subsection{Comparison of ULK2 WT, S507AS750A, or S507D S750D Interaction with AMPK1 $\alpha$}

To find out the reason why ULK2 WT, S507AS750A, or S507DS750D mutant 
was more active than WT, we tried to search for protein that could interact with the S/P space domain of ULK2 (Figure 2(a)). AMPK1 is known to be involved in the dual feedback (positive or negative) regulation of a variety of protein kinases. AMPK1 is a conserved sensor of intracellular energy activated in response to low nutrient availability and environmental stress. Therefore, it was considered as a candidate protein that might interact with S/P space of ULK2. As shown in Figure 2(a), it has been reported that the S/P space domain of ULK2 WT protein interacted with AMPK1. To confirm this observation, we performed coimmunoprecipitation of AMPK1with EGFP ULK2 WT, S507A, S507AS750A, or S507DS750D in HEK293 cells. ULK2 S507AS750A mutant brought down AMPK1 $\alpha$ than WT, S507A, and S507DS705D effectively (Figure 3(a)), while ULK2 S507DS750D did less co-immunoprecipitate with AMPK1 $\alpha$ in HEK 293 cells. Thus, the result suggests that the phosphorylation on Ser507, Ser705 residue of ULK2 inhibits the interaction between ULK2 and AMPK1 $\alpha$.

To confirm the interaction between ULK2 and AMPK1 $\alpha$ again, the confocal microscopy observation was performed with EGFP ULK2 WT, S507A, S507AS750A, or S507DS750D and AMPK1 $\alpha$ antibody in HEK293 cells. As shown in Figure 3(b), the highest PCC value between ULK2 S507AS750A and AMPK1 $\alpha(0.88 \pm$ $0.05 ; \mathrm{N}=5$ ) among them was accounted, while PCC value between ULK2 S507DS750D and AMPK1 was $(0.45 \pm 0.06$; $\mathrm{N}=5)$. Consistent with the above co-immunoprecipitation result (Figure 2(a)), the confocal results also suggest that ULK2 unphosphorylated form (ULK2 S507AS750A) prefers to bind with AMPK1 $\alpha$ than ULK2 phosphorylated form (ULK2 S507DS750D).

To confirm again whether the phosphorylation of ULK2 Ser507 and Ser750 contributes to its subcellular localization in Figure 2(d), we also determined Fn/c value of each ULK construct under the confocal microscopy (Figure 3(b)). EGFP-ULK2 S507AS750A $(0.88 \pm 0.05 ; \mathrm{N}=5 ; \mathrm{p}<0.05)$ was the highest among three (ULK2 WT $(0.68 \pm 0.06 ; \mathrm{N}=5 ; \mathrm{p}<0.05)$ and S507DS750D $(0.45 \pm 0.06 ; \mathrm{N}=5 ; \mathrm{p}<0.05)$. This result also supports the above observation that the phosphorylation on ULK2 Ser507 and Ser750 residue by SGK1 promotes its nuclear localization.

\subsection{The Effect of ULK2 WT, S507AS750A, or S507DS750D on HEK293 Cell Viability}

To access further the effect of ULK2 phosphorylation by SGK1 on cell viability, the EGFP ULK2 WT or its S507A S750A or S507D S750D mutant or EGFP vector was transfected in HEK293 cell and the rate of apoptosis was measured by FACS (Table 1). While EGFP ULK2 S507AS750A mutant showed significantly two times lower an apoptosis rates $(7.16 \% \pm 0.86 \% ; \mathrm{N}=5)$ than ULK2 WT $(11.73 \pm 2.52)$ or ULK2 S507DS750D $(20.16 \pm 1.46 ; \mathrm{N}=5$; $\mathrm{p}<0.05)$ mutant construct, suggesting that the unphosphorylated ULK2 (S507AS750A mutant) contributes the cell survival effectively, while the phosphorylated ULK2 on its Ser507 and Ser750 residue by SGK1 (ULK2 S507DS750D) does not. Conversely, the phosphorylation on ULK2 Ser507 and Ser750 residue by SGK1 promotes cell death (Figure 4 and Table 1). 
(a)

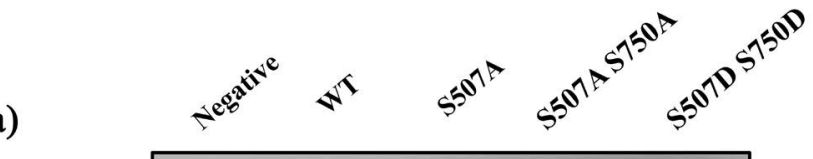

IP : EGFP

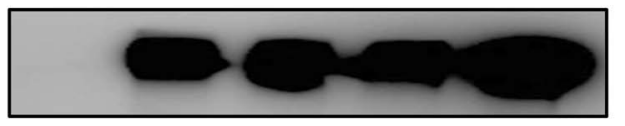

IB : ULK2

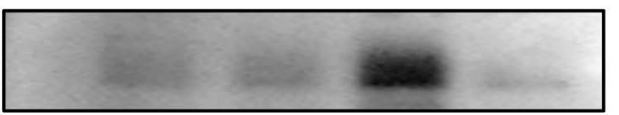

AMPK1 $\alpha$

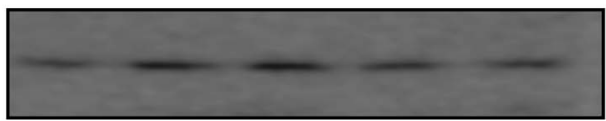

Actin

(b)

ULK2 WT

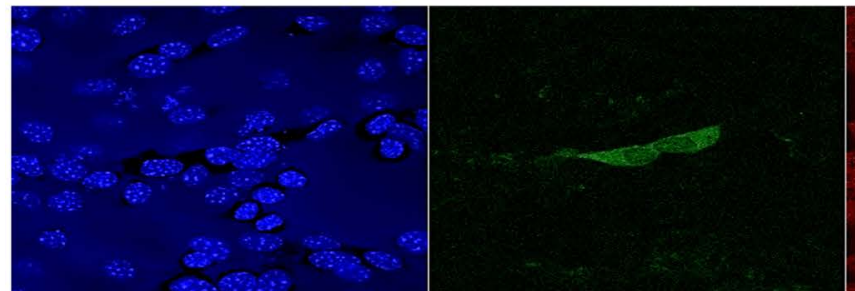

Fn/c: $0.21+/-0.06(N=5)$

ULK2 S507AS750A

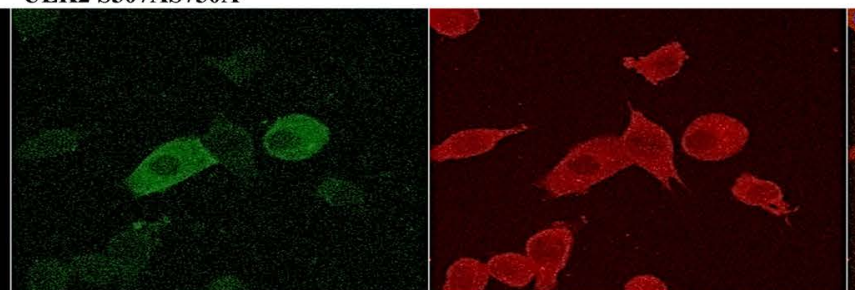

$\mathrm{Fn} / \mathrm{c}: 0.17+/-0.07(\mathrm{~N}=5)$

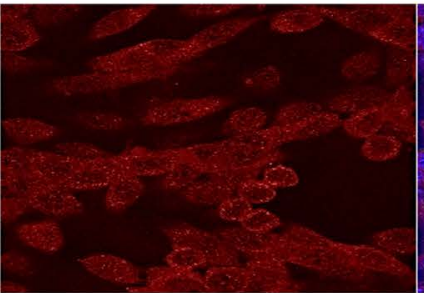

2:

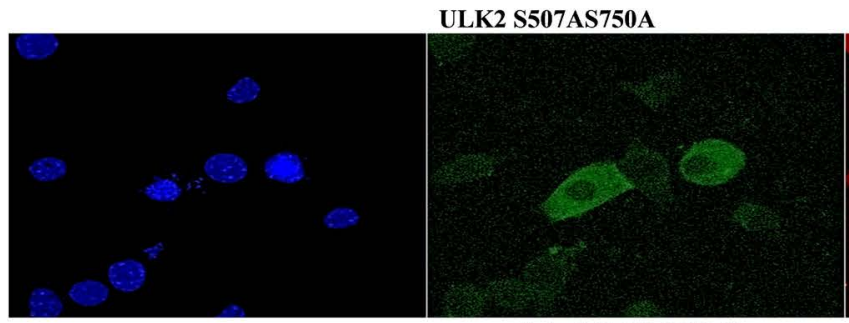

ULK2 S507DS750D

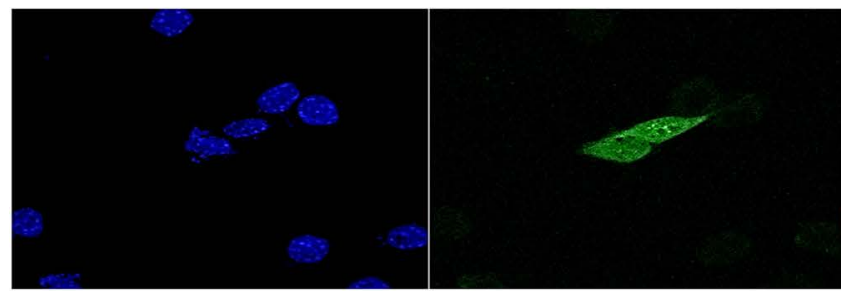

Fn/c: $0.52+/-0.04(\mathrm{~N}=5)$

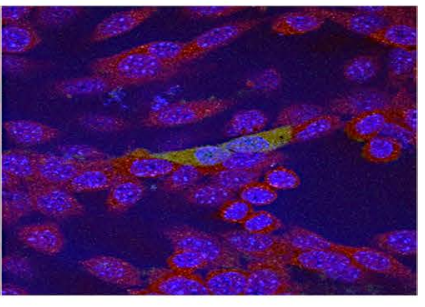

PCC: $0.68+/-0.06(\mathrm{~N}=5)$.

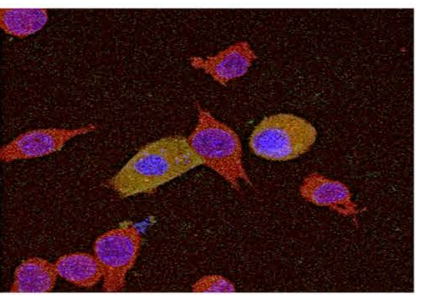

PCC: $0.88+/-0.05(\mathrm{~N}=5)$

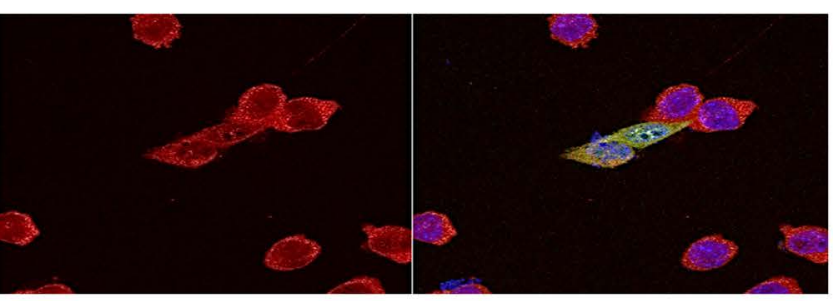

PCC: $0.45+/-0.06(\mathrm{~N}=5)$.

Figure 3. The interaction of ULK2 mutant with AMPK1 depending on Ser507 and 750 phosphorylation. (a) Co-immunoprecipitation of ULK2 WT, S507A, S507AS750A, S507DS750D or with AMPK1. HEK293 cells were transiently transfected with EGFP-ULK2 WT or S750A plasmid. After $48 \mathrm{~h}$, the cells were lysed, total proteins were recollected, and immunoprecipitation was conducted with EGFP Ab. Western blot assays were then conducted with a rabbit ULK2Ab or an anti-AMPK1 $\alpha$ antibody in HEK293 cells. ULK2 S507DS750D (analog of SGK1 phosphorylated UNK2) was less 1/3 brought down AMPK1 $\alpha$ than ULK2 WT or S507AS750A was. To monitor the total amount protein, western bolt with actin antibody was done in the third lane. This is one of four repeated experiment results. (b) Confocal microscopic analysis of transfected EGFP-ULK2 WT or mutant (S750A or S750D) (all constructs are shown in green) with AMPK1 $\alpha$ (red). The transfected EGFP ULK2 WT was merged as yellow color. However, the transfected EGFP-ULK2 S750D or S750A was not merged with AMPK1 $\alpha$ (shown in red). Fn/c value of EGFP-ULK2 S507DS750D (0.45) was the highest among three. DAPI staining represents the nuclear region (blue). PPC between AMPK1 $\alpha$ and ULK2 was $0.68 \pm 0.06(\mathrm{~N}=5)$. DAPI staining represents the nuclear region (blue). The image was taken with the direct immunofluorescence microscopy $(\times 640)$. The Fn/c was indicated under the picture and each PCC was on the right. 


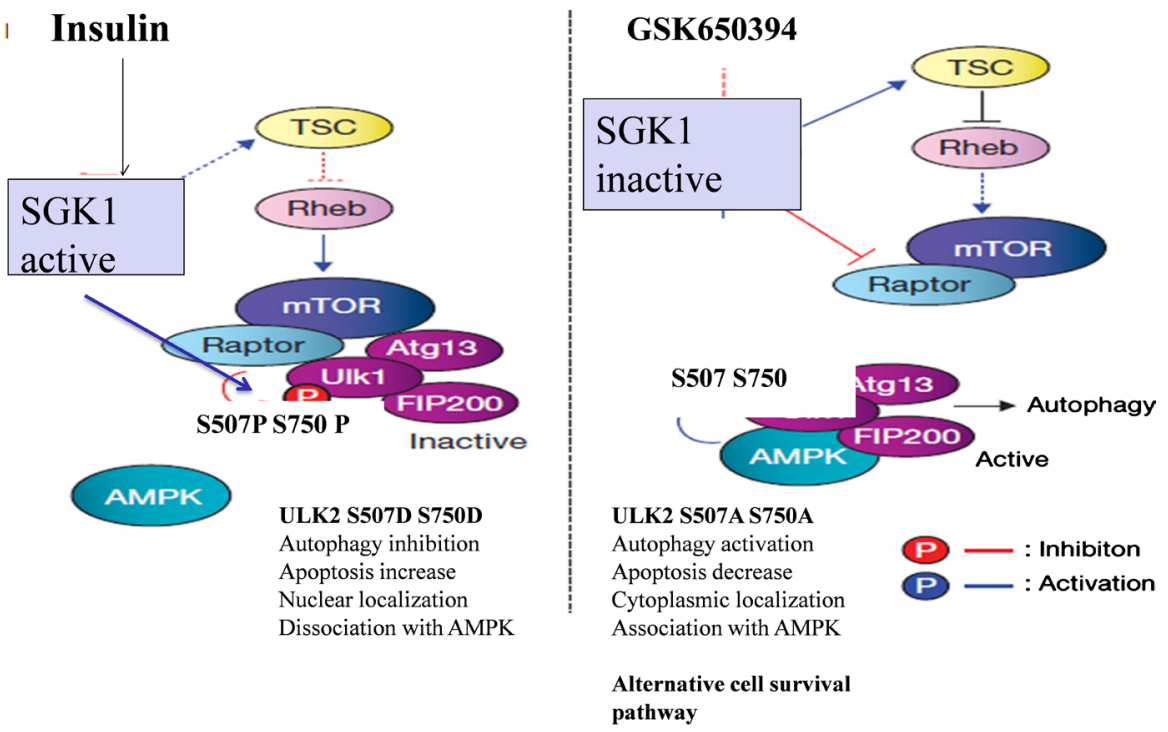

Figure 4. Schematic presentation of regulation on the interaction between ULK2 and AMPK1 by SGK1 phosphorylation. ULK2 WT can be inactivated by SGK1 phosphorylation on its 507 and 750 serine. The functional proteins (such as Atg13-AMPK1 $\alpha$ ) are disassociated. SGK1 activation by insulin inhibits ULK2 autophagy activity through the enhancement of AMPK1 $\alpha$ dissociation, while SGK1 inactivation by GSK650394 [19] promotes ULK2 autophagy activity through the enhancement of AMPK1 $\alpha$ association. From the action mechanism of ULK1 [20] [21], this picture was redraw by replacing it with ULK2. Contrasted with ULK2 S507D S750D (which is an analogue of the phosphorylated ULK2 and is localized into the nuclear and increase apoptosis), ULK2 S507AS750A mutant (which is an analogue of the unphosphorylated ULK2 and is localized into the cytoplasm) can be readily associated with AMPK1 $\alpha$, and thus remaining an active form for the autophagy (decrease apoptosis, more detail, see the discussion section).

Table 1. Comparison of ULK2 cell viability with its WT, S750A or S750D mutant. EGFP ULK2WT or its S507AS750A or S507DS750D mutant or EGFP vector was transfected and the rate of apoptosis was measured by FACS. EGFP ULK2 S507AS750A mutant showed significantly lower an apoptosis rates than ULK2 WT or ULK2 S507DS750D mutant construct.

\begin{tabular}{cc}
\hline ULK2 & Rate of apoptosis [\%] by FACS \\
\hline Non treatment & $11.73 \pm 2.52$ \\
WT & $15.16 \pm 1.76$ \\
S507AS750A & $7.16 \pm 0.86^{*}$ \\
S507DS750D & $20.16 \pm 1.46^{*}$ \\
Vector only & $10.69 \pm 1.75$ \\
& Mean value of 5 repeats \\
\hline
\end{tabular}

${ }^{*} \mathrm{p}$ value less than 0.05 .

In summary, these results support that SGK1 can phosphorylate on Ser507 or Ser750 residue of ULK2, as one of its authentic substrate proteins. As a consequence of SGK1 phosphorylation of two serine residues of ULK2, its autophagy activity and association with AMPK1 $\alpha$ are decreased (Figure 4). Its nuclear localization is also promoted by Ser507 and 750 residue phosphorylation. Our results 
suggest that when growth stimulant is diffused away, resulting in SGK1 inactivation, the activation of ULK2 (by unphosphorylation) occurs as a backup system for cell survival as an alternative cell survival pathway (Figure 4).

\section{Discussion}

Here, we revealed some intriguing features of the mechanism relevant to ULK2 regulation by SGK1 phosphorylation on either Ser507 and/or 750 residues of ULK2 (Figure 1 and Figure 2). We also documented that, in addition to a positive effect of the phosphorylation on both Ser507 and Ser750 of ULK2, a potential effect of autophagy with LC3II binding occurred via its action at an intracellular site in the C-terminus of the kinase mediated by adenosine monophosphate-activated kinase $1 \alpha$ [AMPK1 $\alpha$ ] binding (Figure 3). Based on other reports, we expected that the inhibition of ULK2 activity by Ser507 or Ser750 phosphorylation was agonized or antagonized by selective SGK1 activity modulators (insulin and GSK650394). After treatment with insulin or GSK650394, we observed ULK2WT property change as expected. However, we found that the basal activity of ULK2 S507A/D S750A/D (an analog of unphosphorylated ULK2 by SGK1) was higher than that of ULK2WT or ULK2 S507A/D S750S/D (an analog of phosphorylated ULK2 by SGK1). This observation suggests that the S/P space domain of ULK2 near the serine residue 507 and Ser750 seems to be assigned to regulate its function by unknown mechanism beyond phosphorylation modification such as protein-protein interaction with AMPK1 $\alpha$ or membrane [18] [20]. With regard to ULK2, its interaction with AMPK1 is regulated competitively by SGK1-mediated phosphorylation of serine 507 and/or Ser750 residue within AMPK1 binding domain [20] [21]. ULK2 contains a consensus sequence for protein-Ser750 phosphorylation by SGK1 within the AMPK1 binding domain (Figure 2(a)). However, mutation of this serine residue, which is a prospective phosphorylation site within this domain in mutant ULK2 S750A, affected autophagy-dependent potentiation. Additionally, ULK2 $\left({ }^{349} \mathrm{tddfvlv}^{356}\right)$ binding by LC3I is modified by phosphorylation on Ser750 (Figure 2(c)). Although we found that AMPK1 could interact with ULK2, other unknown proteins could also regulate ULK2 function through protein-protein interaction with its S/P space domain.

In this regard, AMPK1 binding site in its C-termini seems to be involved in autophagy-induced conformational changes. It is likely that both Ser507 and/or Ser750 of the S/P space domain can induce a conformational change in ULK2 protein and increased its kinase activity as both ULK2 S507AS750A (such as ULK2 mutant which is regardless of the phosphorylation) are more active than ULK2 WT. Interestingly, the autophagy of ULK1, ULK2's closest relatives, seems to be regulated at the corresponding region by AMPK1 $\alpha$ through SGK1 phosphorylation [1] [2] [3] [4]. Thus, phosphorylation on either Ser507 and/or Ser750 of ULK2 by SGK1 might inhibit its association with AMPK1 complex. Because AMPK1 is a conserved sensor of intracellular energy activated in re- 
sponse to low nutrient availability and environmental stress, the negative effect of SGK1 phosphorylation on the binding between ULK2 and AMPK1 suggests that SGK1 can antagonize AMPK1 $\alpha$ for the regulation of ULK2. As for ULK1 (a similar kinase of ULK2), it has been suggested that its activity is tightly controlled by autophagy-dependent negative feedback mechanisms in which AMPK1 plays a role. ULK1 has been suggested to be unusual case among plasma membrane autophagy permeable kinases with moderate to high autophagy in that it is potentiated by increases in intracellular autophagy concentration. However, the deleterious increase in intracellular autophagy resulting from starvation-activated autophagy entry appears to be prevented by autophagy-dependent negative feedback (Figure 1). Results of the present study provide clues suggesting the importance of ULK2 Ser507 $\left({ }^{502} \mathrm{RsRnsSg}^{508}\right)$ and Ser750 residue $\left({ }^{745} \mathrm{RtRttSv}{ }^{751}\right)$ in the regulation of self-kinase in response to stress, AMPK1 $\alpha$ binding, and its subcellular localization to plasma membrane via SGK1phosphorylation (Figure 2(d) and Figure 3(b)).

Interestingly, we noticed the presence of SGK1 substrate motif Ser 774 $\left({ }^{770} \mathrm{rtrmfsagptg}^{780}\right)$ in ULK1 [4]. The presence of this motif suggests that ULK1 might also be one of SGK1 substrate proteins. Similar to ULK2, it is assumed that phosphorylation on ULK1 Ser774 residue by SGK1 also inhibits its autophagy activity [1] [2] [3] [4]. The putative binding site in ULK2 by AMPK1 $\alpha$ is assumed from ULK1 binding site of AMPK1 $\alpha$ based on amino acid sequence homology comparison (Figure 2(a)) [4]. Because Ser750 residue phosphorylation by SGK1 inhibits AMPK1 binding with ULK2, the phosphorylation on Ser750 is one of antagonistic effects of SGK1 survival signal in response to autophagy. Although we documented that ULK2 also interacted with AMPK1 $\alpha$ (Figure 2(a)), its exact binding site for AMPK1 $\alpha$ should be determined experimentally.

Thus, phosphorylation of SGK1 on ULK1/2 may be equivalent to that of mTORC1 which inhibits both ULK1 and ULK2 by the phosphorylation. Moreover, our results suggest that ULK2 Ser750 residue phosphorylation by SGK1 also contributes to autophagy-related effect or its regulation through interaction between AMPK1 $\alpha$ and ULK2. Until now, SGK1 has not been reported to have association with mammalian autophagy. In this regard, SGK1 might essentially have the similar function as mTORC1 to inactivate ULK1/2. To control both cell autophagy and survival, these two kinases (mTORC1 and SGK1) appear to work together in a synergistic fashion. ULK2 Ser729 residue in ( ${ }^{721} \mathrm{kavlftvgSpphs}^{733}$ ) which is close to ULK2 Ser750 is a putative phosphorylation site by mTORC1 [1] [2] [3] [4]. Therefore, it is also worth noting that both SGK1 and mTORC1 are involved in the regulation of cell survival and autophagy synergistically.

Previously, we have documented that ULK2 contains PY-NLS which binds to karyopherin $\beta 2$ for autophagy $\left({ }^{783}\right.$ gaeaapslryvpy $\left.{ }^{795}\right)$ in its SP space domain, but not ULK1. Due to change of ULK2 conformation by SGK1 phosphorylation on its Ser750 residue $\left({ }^{75} \mathrm{RtRttSv}{ }^{751}\right)$, the PY-NLS which is available to karyopherin 
$\beta 2$ may eventually help transport it into the nuclear. However, the specific function of ULK2 in the nuclear for autophagy is currently unclear. Analysis of amino acid sequence did not reveal the presence of any recognizable import or export sequence. Its localization may be indirectly regulated through association with binding proteins. ULK2 is located predominantly in the nuclei, although it is also present in the cytosol. However, the mechanism by which ULK2 localization is controlled remains unclear. Previously, it has been demonstrated that ULK2 is modulated through phosphorylation at its 1027 serine residue by Protein Kinase A (PKA), one of its authentic substrate proteins [11]. As a consequence of PKA-phosphorylation on both Ser 468 and Ser1027 of ULK2, its self-kinase activity on its 278 - 351 fragment and autophagy were reduced, coupled with an increase in sensitivity to starvation and stress, a promotion of its nuclear localization [our unpublished data]. Interestingly, near the Ser1027 residue region, putative NES sequence form CRM1 binding site was noticed. The phosphorylation on Ser1027 residue of ULK2 by PKA may interfere its association with CRM1, resulting in ULK2's nuclear localization. Thus, ULK2 subcellular localization seems to be regulated by its specific serine/threonine residue phosphorylation by both SGK1 and PKA, similar with Figure 2(d) and Figure 3(b). Many ligands such as peptide hormones frequently conduct signaling via SGK1 transduction pathways to activate downstream effectors. The concept of a parallel transduction pathway involving SGK1 is gaining increasing credibility among researchers. Indeed, it is now known that a number of signaling proteins have consensus sequence (R-X-R-X-X-(S/T)- $\Phi)$ and that they can be phosphorylated by SGK1 kinase to perform critical roles in the regulation of signaling functions [12] [13] [14]. Evidence of such regulatory mechanisms via kinase phosphorylation, however, has yet to be definitively reported. Results of this study should facilitate further investigations into this matter. Akt kinase may phosphorylate the serine/threonine residue in the consensus sequence of (R-X-R-X-X-(S/T)- $\Phi)$ which can be phosphorylated by SGK1 [12] [13] [14]. Until now, several SGK1 substrates have been characterized. These results indicate that SGK1 plays a role, in concert with Akt1, in propagating effects of PI3K, including promotion of cell-cycle progression and consequently cell survival. Ser507 and 750 of ULK2 can also be phosphorylated by Akt kinase which is a key protein kinase for cell survival [14]. Because ULK2 is involved in cell apoptosis, it seems to be reasonable that both SGK1 and Akt1 are antagonistic to ULK2 role by its Ser507 or Ser750 residue phosphorylation. ULK1 also has a putative SGK1 phosphorylation site on Ser775 in $\left({ }^{770} \mathrm{RtRmfSA}{ }^{776}\right)$ which is also characterized as AMPK1a phosphorylation site [1] [2] [3] [4]. Therefore, similar to ULK2 in this paper, SGK1 phosphorylation on Ser775 of ULK1 might also function as an antagonistic to AMPK1 $\alpha$ (Figure 4). However, it remains to be characterized whether ULK1 phosphorylation by SGK1 inhibits its autophagy activity. Phosphorylation on Ser750 residue of ULK2 by SGK1 (as its authentic substrate protein) is also one of regulatory points to activate its autophagy functions. It has been reported 
that mTOR1 phosphorylation on Ser729 in (kavlftvgSpphs) of ULK2 also enhances ULK2's autophagy activity [1] [2] [3] [4].

In summary, our results clearly demonstrate that ULK2 is regulated through phosphorylation of its either Ser507 or Ser750 residue by SGK1 as one of its authentic substrate proteins. As a consequence of phosphorylation of Ser507 and/or 750 residues of ULK2 by SGK1, its phosphorylation and association with AMPK1 $\alpha$ were decreased, coupled with an increase of an antagonistic effect on its autophagy (Figure 4, Table 1). SGK1, similar to Akt kinase as a downstream effector of PI3K-PDK1 signal pathway for cell survival [13] [19], can inhibit ULK2 autophagy activity by phosphorylation as its authentic substrate proteins until they are active (Figure 4). Therefore, cell survival and autophagy are not antagonistic, but agonistic for cell survival in the transformed cell line [14] [15] [16].

\section{Capsule}

Background: UNC-21-like kinase 2 (ULK2) harbors putative phosphorylation residues Ser507and Ser750 by serum- and glucocorticoid-induced kinase 1 (SGK1).

Result: SGK1 phosphorylates Ser507 andSer750 residues of ULK2.

Conclusion: PhosphorylationofSer507 and Ser750 residues of ULK2 by SGK1inhibits its autophagy activity.

Significance: Activation of ULK2autophagyis regulated as an alternative cell survival pathway during SGK1 inactivation.

\section{Acknowledgements}

This work was supported by a Korean Research Foundation grant (BK21 plus and NRF-2016R1D1A3B03934269) to S. S. Kang. Shin is Korean Research fellowship recipients (NRF-2014R1A1A2009622).

\section{Conflicts of Interest}

The authors declare no conflicts of interest regarding the publication of this paper.

\section{References}

[1] Corona Velazquez, A.F. and Jackson, W.T. (2018) So Many Roads: The Multifaceted Regulation of Autophagy Induction. Molecular and Cellular Biology, 38, pii: e00303-18. https://doi.org/10.1128/MCB.00303-18

[2] Wang, B. and Kundu, M. (2017) Canonical and Noncanonical Functions of ULK/Atg1. Current Opinion in Cell Biology, 45, 47-54. https://doi.org/10.1016/j.ceb.2017.02.011

[3] Alers, S., Löffler, A.S., Wesselborg, S. and Stork, B. (2012) The Incredible ULKs. Cell Communication and Signaling, 10, 7. https://doi.org/10.1186/1478-811X-10-7

[4] Yan, J., Kuroyanagi, H., Tomemori, T., Okazaki, N., Asato, K., Matsuda, Y., Suzuki, Y., Ohshima, Y., Mitani, S., Masuho, Y., Shirasawa, T. and Muramatsu, M. (1999) 
Mouse ULK2, a Novel Member of the UNC-51-Like Protein Kinases: Unique Features of Functional Domains. Oncogene, 18, 5850-5859.

https://doi.org/10.1038/sj.onc.1202988

[5] Zhou, X., Babu, J.R., da Silva, S., Shu, Q., Graef, I.A., Oliver, T., Tomoda, T., Tani, T., Wooten, M.W. and Wang, F. (2007) Unc-51-Like Kinase 1/2-Mediated Endocytic Processes Regulate Filopodia Extension and Branching of Sensory Axons. Proceedings of the National Academy of Sciences of the United States of America, 104, 5842-5847. https://doi.org/10.1073/pnas.0701402104

[6] Lee, E.J. and Tournier, C. (2011) The Requirement of Uncoordinated 51-Like Kinase 1 [ULK1] and ULK2 in the Regulation of Autophagy. Autophagy, 7, 689-695. https://doi.org/10.4161/auto.7.7.15450

[7] Wang, B., Iyengar, R., Li-Harms, X., Joo, J.H., Wright, C., Lavado, A., Horner, L., Yang, M., Guan, J.L., Frase, S., Green, D.R., Cao, X. and Kundu, M. (2018) The Autophagy-Inducing Kinases, ULK1 and ULK2, Regulate Axon Guidance in the Developing Mouse Forebrain via a Noncanonical Pathway. Autophagy, 14, 796-811. https://doi.org/10.1080/15548627.2017.1386820

[8] Sumitomo, A., Yukitake, H., Hirai, K., Horike, K., Ueta, K., Chung, Y., Warabi, E., Yanagawa, T., Kitaoka, S., Furuyashiki, T., Narumiya, S., Hirano, T., Niwa, M., Sibille, E., Hikida, T., Sakurai, T., Ishizuka, K., Sawa, A. and Tomoda, T. (2018) Ulk2 Controls Cortical Excitatory-Inhibitory Balance via Autophagic Regulation of p62 and GABAA Receptor Trafficking in Pyramidal Neurons. Human Molecular Genetics, 27, 3165-3176. https://doi.org/10.1093/hmg/ddy219

[9] Ran, M., Li, Z., Cao, R., Weng, B., Peng, F., He, C. and Chen, B. (2018) miR-26a Suppresses Autophagy in Swine Sertoli Cells by Targeting ULK2. Reproduction in Domestic Animals, 53, 864-871. https://doi.org/10.1111/rda.13177

[10] Jatana, N., Ascher, D.B., Pires, D.E.V., Gokhale, R.S. and Thukral, L. (2019) Human LC3 and GABARAP Subfamily Members Achieve Functional Specificity via Specific Structural Modulations. Autophagy, 14, 1-17. https://doi.org/10.1080/15548627.2019.1606636

[11] Shin, S.H., Lee, E.J., Chun, J., Hyun, S. and Kang, S.S. (2015) ULK2 Ser 1027 Phosphorylation by PKA Regulates Its Nuclear Localization Occurring through Karyopherin Beta 2 Recognition of a PY-NLS Motif. PLoS ONE, 10, e0127784. https://doi.org/10.1371/journal.pone.0127784

[12] Lang, F. and Shumilina, E. (2013) Regulation of Ion Channels by the Serum- and Glucocorticoid-Inducible Kinase SGK1. The FASEB Journal, 27, 3-12. https://doi.org/10.1096/fj.12-218230

[13] Lang, F. and Cohen, P. (2001) Regulation and Physiological Roles of Serum- and Glucocorticoid-Induced Protein Kinase Isoforms. Science s STKE, 2001, re17. https://doi.org/10.1126/stke.2001.108.re17

[14] Kobayashi, T., Deak, M., Morrice, N. and Cohen, P. (1999) Characterization of the Structure and Regulation of Two Novel Isoforms of Serum- and Glucocorticoid-Induced Protein Kinase. Biochemical Journal, 344, 189-197. https://doi.org/10.1042/bj3440189

[15] Chun, J., Kwon, T., Kim, D.J., Park, I., Chung, G., Lee, E.J., Hong, S.K., Chang, S.I., Kim, H.Y. and Kang, S.S. (2003) Inhibition of Mitogen-Activated Kinase Kinase Kinase 3 Activity through Phosphorylation by the Serum- and Glucocorticoid-Induced Kinase 1. The Journal of Biochemistry, 133, 103-108. https://doi.org/10.1093/jb/mvg010

[16] Chun, J., Kwon, T., Lee, E., Suh, P.G., Choi, E.J. and Sun Kang, S. (2002) The $\mathrm{Na}[+] / \mathrm{H}[+]$ Exchanger Regulatory Factor 2 Mediates Phosphorylation of Serum- 
and Glucocorticoid-Induced Protein Kinase 1 by 3-Phosphoinositide-Dependent Protein Kinase 1. Biochemical and Biophysical Research Communications, 298, 207-215. https://doi.org/10.1016/S0006-291X(02)02428-2

[17] Brunet, A., Park, J., Tran, H., Hu, L.S., Hemmings, B.A. and Greenberg, M.E. (2001) Protein Kinase SGK Mediates Survival Signals by Phosphorylating the Forkhead Transcription Factor FKHRL1 [FOXO3a]. Molecular and Cellular Biology, 21, 952-965. https://doi.org/10.1128/MCB.21.3.952-965.2001

[18] Zhou, C., Gu, J., Zhang, G., Dong, D., Yang, Q., Chen, M.B. and Xu, D. (2017) AMPK-Autophagy Inhibition Sensitizes Icaritin-Induced Anti-Colorectal Cancer Cell Activity. Oncotarget, 8, 14736-14747.

https://doi.org/10.18632/oncotarget.14718

[19] Inoue, K., Sakuma, E., Morimoto, H., Asai, H., Koide, Y., Leng, T., Wada, I., Xiong, Z.G. and Ueki, T. (2016) Serum- and Glucocorticoid-Inducible Kinases in Microglia. Biochemical and Biophysical Research Communications, 478, 53-59. https://doi.org/10.1016/j.bbrc.2016.07.094

[20] Alers, S., Löffler, A.S., Wesselborg, S. and Stork, B. (2012) Role of AMPK-mTOR-Ulk1/2 in the Regulation of Autophagy: Cross Talk, Shortcuts, and Feedbacks. Molecular and Cellular Biology, 32, 2-11. https://doi.org/10.1128/MCB.06159-11

[21] Khan, S.H. and Kumar, R. (2012) Role of an Intrinsically Disordered Conformation in AMPK-Mediated Phosphorylation of ULK1 and Regulation of Autophagy. Molecular BioSystems, 8, 91-96. https://doi.org/10.1039/C1MB05265A 